\title{
Methylmercury Interactions With Gut Microbiota and Potential Modulation of Neurogenic Niches in the Brain
}

\author{
Daniel V. Pinto ${ }^{1}$, Ramon S. Raposo ${ }^{2}$, Gabriella A. Matos ${ }^{1}$, Jacqueline I. Alvarez-Leite ${ }^{3}$, \\ João O. Malva ${ }^{4}$ and Reinaldo B. Oriá ${ }^{1 *}$ \\ ${ }^{1}$ Laboratory of Tissue Healing, Ontogeny and Nutrition, Department of Morphology, School of Medicine, Institute of \\ Biomedicine, Federal University of Ceara, Fortaleza, Brazil, ${ }^{2}$ Experimental Biology Core, University of Fortaleza, Fortaleza, \\ Brazil, ${ }^{3}$ Department of Biochemistry and Immunology, Institute of Biological Sciences, Federal University of Minas Gerais, \\ Belo Horizonte, Brazil, ${ }^{4}$ Center for Innovative Biomedicine and Biotechnology (CIBB), Faculty of Medicine, Institute of \\ Pharmacology and Experimental Therapeutics, Institute for Clinical and Biomedical Research (iCBR), University of Coimbra, \\ Coimbra, Portugal
}

Keywords: methylmercury, neurogenesis, brain, intestinal microbiota, neurodegenerative diseases, gut dysbiosis

\section{INTRODUCTION}

Mercury $(\mathrm{Hg})$ is a well-recognized biohazard for the nervous system. Methylmercury (MeHg) is an organic methylated form of $\mathrm{Hg}$, highly toxic to humans, targeting the brain, as $\mathrm{MeHg}$ is rapidly absorbed, and easily reaches and crosses the blood-brain barrier (Takahashi et al., 2017).

OPEN ACCESS

Edited by:

Vittorio Calabrese,

University of Catania, Italy

Reviewed by:

María A. García

University of Concepcion, Chile

${ }^{*}$ Correspondence:

Reinaldo B. Oriá

reinaldo70.oria@gmail.com

Specialty section:

This article was submitted to

Neuroenergetics, Nutrition and Brain

Health,

a section of the journal

Frontiers in Neuroscience

Received: 26 June 2020

Accepted: 24 September 2020

Published: 03 November 2020

Citation:

Pinto DV, Raposo RS, Matos GA,

Alvarez-Leite Jl, Malva JO and Oriá RB

(2020) Methylmercury Interactions

With Gut Microbiota and Potential

Modulation of Neurogenic Niches in

the Brain. Front. Neurosci. 14:576543.

doi: 10.3389/fnins.2020.576543
Neurological symptoms may vary from acute motor and visual effects to marked behavioral and psychiatric alterations. At higher neurotoxic levels, $\mathrm{MeHg}$ can lead to irreversible coma and, ultimately, death. It has been highlighted that $\mathrm{MeHg}$ long-term and low-grade toxicity may be associated with neurodegenerative disorders and perhaps a direct causality for Alzheimer's disease (Siblerud et al., 2019).

Although $\mathrm{MeHg}$ harmful effects to the brain have been thoroughly documented in the literature, such as increased oxidative stress and mitochondrial dysfunction, halted glutamate uptake by astrocytes and overt glutamate excitotoxicity, and activation of neuronal apoptosis cascades (Antunes dos Santos et al., 2016), less is known how MeHg affects the hippocampal neurogenic niche.

Hence, in this opinion paper, we summarize up-to-date literature addressing $\mathrm{MeHg}$ effects on the intestinal microbiota, a key player influencing $\mathrm{MeHg}$ bioavailability and $\mathrm{MeHg}$ induction of intestinal dysbiosis (and vice-versa), and related intricate mechanisms during homeostasis and disease states. In addition, we discuss possible ways how $\mathrm{MeHg}$ may affect hippocampal neurogenesis and the potential lasting consequences for brain neurodegeneration.

\section{INTESTINAL MICROBIOTA IS AFFECTED BY MeHg}

In mammals, the intestinal microbiota is first acquired either by contact with maternal skin (if a cesarean labor) or directly maternal microbiota transfer (if by vaginal labor) immediately after birth (Shao et al., 2019) and through breast milk feeding (Pannaraj et al., 2017). The intestinal microbiota diversity is highly dynamic in the first years of post-natal life, until it reaches its "adulthood-like" characteristics in early childhood (Oriá et al., 2018). The first 2-years of life is also a time window for important early post-natal brain plasticity events, such as active synaptogenesis, myelination, and neurogenesis (Lebel and Deoni, 2018). Moreover, enteric infections may prevail early in life when individuals are exposed to enteric pathogens and pathogenic bacteria, especially in unprivileged settings of the developing world, where poor sanitation and hygiene much often occurs 
(Oriá et al., 2016). In the first post- natal years, the brain is particular vulnerable to environmental toxicants, as well (Rodier, 1995).

The intestinal absorption of $\mathrm{MeHg}$ may, indeed, be modulated by the intestinal microbiota and by a healthy (intact) intestinal barrier function. Impaired intestinal barrier function has been associated with increased $\mathrm{MeHg}$ intestinal absorption (Zhao et al., 2020), altered blood-brain barrier, and disrupted VEGF signaling (Takahashi et al., 2017).

A "leaky gut" may markedly increase circulating LPS, trigger peripheral inflammation and oxidative stress that can reach the central nervous system, leading to brain neuroinflammation, with increased microglia priming and activation (GarcíaDomínguez et al., 2018). Circulating LPS levels in peripheral blood have been associated with a reduction in neurogenesis and mild cognitive deficit in experimentally Alzheimer's disease model (Valero et al., 2014).

$\mathrm{MeHg}$-driven intestinal injury may be protected by orallygiven Lactobacillus, a known bacteria that can generate short- chain fatty acids (SCFAs) in the large intestine (Jiang et al., 2018). SCFAs are important for microglia maturation and function (Wenzel et al., 2020) and reduced SCFA production by the gut microbiota has been related to neurodegenerative diseases (Zhang et al., 2017). Of note, fecal samples from human APOE4 carriers (individuals at higher risk to develop Alzheimer's disease) are significantly less enriched in Ruminococcacea family of anaerobes associated with fiber fermentation and production of SCFAs (Tran et al., 2019).

One form of modulation is the demethylation of $\mathrm{MeHg}$ into inorganic elements by the intestinal microbiota, which reduces $\mathrm{Hg}$ solubility in the tissue and hence bioavailability to the brain. The upregulation in Peptococcaceae family in the rat gut has been found to promptly demethylate $\mathrm{MeHg}$ and increase fecal excretion of $\mathrm{Hg}$ elements (Lin et al., 2020). Fecal excretion is one important way to eliminate $\mathrm{Hg}$ and prevent $\mathrm{MeHg}$ intoxication (Clarkson and Magos, 2006). Two-genes clusters, hgcA and hgcB, are required for mercury methylation by gut bacteria (Parks et al., 2013). Several hgcAB-encoding bacterial strains may affect gut net $\mathrm{Hg}$ bacterial methylation (Zhang et al., 2019).

Another microbiota-directly related event is the modulation of bacterial siderophores, that can bind to $\mathrm{Hg}$ molecules, acting as chelators, forming insoluble complexes and facilitating their excretion in the feces (Schalk et al., 2011). Such effect may modulate the bioavailability of other metals such as iron and zinc, brain trophic key nutrients, that may also regulate intestinal bacterial overgrowth (Lopez and Skaar, 2018).

Oral administration of $\mathrm{HgCl} 2$ to female mice can also induce alterations in the intestinal microbiota homeostasis increasing the Firmicutes/Bacteroidetes ratio (Ruan et al., 2019). This alteration in Firmicutes/Bacteroidetes ratio is also seen with the use of antibiotics (Indiani et al., 2018), immunosuppressive agent or even intestinal chronic diseases (Bhat et al., 2017). This ratio has been used as a proxy of an unhealthy gut, being positively correlated with gut dysbiosis and obesity (Leocádio et al., 2020). Furthermore, it has been associated with aging and Alzheimer's disease (Hoffman et al., 2017).
Studies with $\mathrm{MeHg}$ intoxication in 8-week old mice have shown reduced Firmicutes and increased Bacteroidetes numbers compared with non-intoxicated controls (Zhang et al., 2019). In contrast to the metagenomic data from Zhang and colleagues, Lie et al. studies have found that $\mathrm{MeHg}$-orally exposed young rats $(10 \mu \mathrm{g} / \mathrm{Kg})$ showed altered intestinal microbiota with reduced relative abundance of Bacteroidetes and Protobacteria and increases in Firmicutes (Lin et al., 2020). This discrepancy between both studies may be due to different animals and age groups used.

The increase ratio of Bacteroidetes and Firmicutes in young animals may be relevant to hippocampal development and neurogenesis, as the first week of life in rodents are an important time window of brain plasticity. Studies with germ-free mice suggest that a time window early in life is when gut microbes are more modulatory in disturbing hippocampal neurogenesis (Cerdó et al., 2020).

Another relevant alteration in the intestinal microbiota, that has been reported, is the increase in Akkermansia from stools of MeHg-exposed pregnant humans (Rothenberg et al., 2016). These bacteria have been associated with reduced intestinal barrier function and loss of the intestinal mucus layer (Yoshihara et al., 2020). Impairment of the intestinal mucus layer increases the risk for gut dysbiosis, intestinal inflammation, and gut-toblood pathogenic bacteria translocation (Bergstrom et al., 2010), which may be facilitated by $\mathrm{MeHg}$-induced intestinal injury (Zhao et al., 2020).

\section{MeHg EFFECTS ON HIPPOCAMPAL NEUROGENESIS. POTENTIAL MODULATION BY THE GUT MICROBIOTA?}

The hippocampus is critical for memory and learning. These processes rely on complex and intricate neuronal circuitry, involving dentate gyrus granular, CA3 and CA1 pyramidal cell synapses (excitatory trisynaptic neuronal circuit) (Beer et al., 2018). The former neurons are continued renewed at the hippocampal subgranular zone (SGZ) neurogenic niche, which harbors neural stem cells (NSC). The hippocampal neurogenic niche is more active in childhood and may be affected by early-life adverse events with lasting effects (Cohen et al., 2016).

NSC undergoes three maturational stages before reaching full maturity. Stage 1: containing radial-like NSC (GFAP, nestin, and SOX2 positive cells); stage 2, containing transition cells/progenitor cells type 2 a (GFAP, nestin, and SOX 2 positive cells) and type $2 \mathrm{~b}$ (nestin, DCX, Neuro-D, and Prox 1 positive cells) and stage 3 (DCX, Neuro-D, and Proxl positive cells). After stage 3, NSCs reach neuronal maturity by the extension of dendritic and axon processes and functional synaptic activity into neural circuitry (calretin, NeuN, Neuro-D, and Prox1 positive cells) (Kempermann et al., 2015).

$\mathrm{MeHg}$ effects on hippocampal neurogenesis have been mostly studied in the first week of post-natal life in animal models when there is active hippocampal neurogenesis. $\mathrm{MeHg}$ exposure reduces $21 \%$ of the total volume of DNA in the hippocampus, $16 \%$ of the total positive Brdu cells in the granular layer and $50 \%$ 
of cells in the hilus, 14 days after the $5.0 \mu \mathrm{g} / \mathrm{g}$ s.c. injection on the post-natal day (PN) 7 (Falluel-Morel et al., 2007). Kim et al. (2019), also found a 57\% reduction in the number of positive Ki67 cells in the dentate gyrus in 7-week-old Sprague-Dawley rats, 35 days after $5.0 \mu \mathrm{g} / \mathrm{g} \mathrm{MeHg}$ injection (Kim et al., 2019).

A reduction of $22 \%$ of hilus cells and $27 \%$ of granular layer cells, without affecting CA1 to CA3 hippocampal fields, after the $\mathrm{MeHg}$ challenge, has been found in mice, suggesting that $\mathrm{MeHg}$ preferentially affects SGZ's NSC (Sokolowski et al., 2013). Besides, primary cultures of NSC following exposure to $\mathrm{MeHg}$ showed reduced expression of $\mathrm{NADH}$ dehydrogenase and cytochrome $\mathrm{B}$ (Bose et al., 2012), supporting a pro-apoptotic effect of $\mathrm{MeHg}$ in NSC cells. A double immunolabeling of dentate gyrus SOX2 and cleaved caspase 3 cells induced by $\mathrm{MeHg}$ intoxication further suggests the direct target of $\mathrm{MeHg}$ to early hippocampal NSCs (Falluel-Morel et al., 2007).

Falluel-Morel work also documented a decrease in the extracellular signaling of ERK1/2, required for the transition from $\mathrm{Gl}$ to $\mathrm{S}$ phase and inhibition of Bmi-1 gene expression, responsible for controlling the self-renewal potential of NSC. Genes linked to cell senescence have also been shown to be altered by $\mathrm{MeHg}$, such as increased expression levels of HP1- $\gamma$ and HMGA-1 (Falluel-Morel et al., 2007). Long-term antibiotic therapy that disrupts intestinal microbiota homeostasis with increased number of conditionally pathogenic enterobacteria, E. coli, Clostridium, Staphylococcus spp., and hemolytic bacteria, could affect redox-sensitive transcription factor HIF1 $\alpha$ and ERK1/2 MAP kinase and intestinal barrier function (Holota et al., 2019).

Interestingly, early life stress, such as social isolation, can impact neurogenesis and the intestinal microbiota. Socially isolated rats early in life show significantly fewer BrdU/NeuN positive cells in the dentate gyrus than controls and altered microbiota composition with increases in Actinobacteria and decreases in the class Clostridia (Dunphy-Doherty et al., 2018). It would be interesting to know whether these alterations may be further affected by early- life mercury intoxication.

One possible crosstalk pathway between intestinal microbes and the brain may involve the vagal nerve. Vagotomy has been implicated in impaired hippocampal neurogenesis and BDNF levels (O'Leary et al., 2018). Importantly, mercury intoxication has been implicated in disturbed vagal nerve function (Simões et al., 2016).

The better understanding how the MeHg-altered gut microbiota affects the hippocampal neurogenic niche, by tracking neurogenic biomarkers during NSC maturation in critical time windows of brain development, is in most need for novel therapeutic strategies to ameliorate these deleterious effects that may have lasting consequences for human health.

\section{CONCLUSION AND PERSPECTIVES}

This opinion paper discussed the role of the intestinal microbiota on $\mathrm{MeHg}$ neurointoxication with potential consequences for the hippocampal neurogenic niche. Novel breakthrough findings are much supportive of human neurogenesis even in elderly (Boldrini et al., 2018), which was not universally accepted (Sorrells et al., 2018). Activity of NSCs and renewal of hippocampal neurons even in adulthood may be a protective/preventive factor against neurodegenerative diseases. New available neuroimaging tools to study the human brain neurogenesis may shed light to $\mathrm{MeHg}$ deleteriously effects on dynamic processes occurring in the hippocampal neurogenic niche during lifespan.

Accumulating evidence implies the gut-brain axis as a pathway for $\mathrm{MeHg}$ harmful neurotoxic effects and a potential factor for later neurodegenerative disorders. The $\mathrm{MeHg}$ may induce a hormesis-related neuronal toxicity. Hormesis is an important redox dependent aging-associated neurodegenerative/ neuroprotective issue (Calabrese et al., 2010). The use of antioxidants, such as plant polyphenols (Calabrese et al., 2010; Leri et al., 2020) and protective nutrients (Oria et al., 2020) may be beneficial in reducing the $\mathrm{MeHg}$-driven neuroinflammatory state and associated cell death with the interplay of the intestinal microbiota. Further research is warranted to elucidate the fine molecular mechanisms and NSC biomarkers during lifelong hippocampal neurogenesis and how they are affected by $\mathrm{MeHg}$. Such findings may contribute to health policies in highly endemic $\mathrm{MeHg}$ intoxication and poor sanitation settings.

\section{AUTHOR CONTRIBUTIONS}

All authors have read and approved the manuscript. All authors have equally contributed to the opinion paper.

\section{ACKNOWLEDGMENTS}

The authors would like to thank FEDER-CENTRO 2020- CENTRO-01-0145-FEDER-000012 (HealthyAging 2020) and COMPETE and FCT (POCI-01-0145-FEDER029221 and UIDB/04539/2020), Pest-C/SAU/UI3282/2013-2014 and CNC.IBILI UID/NEU/04539/2013 with national funds PT2020/COMPETE 2020 and FCT/FUNCAP (POCTI-FEDER02/SAICT/2017/31699), Brazilian CAPES-PROCAD (071/2013 \# 88881.068408/2014-01) and CNPq-PVE grants.

\section{REFERENCES}

Antunes dos Santos, A., Appel Hort, M., Culbreth, M., López-Granero, C., Farina, M., Rocha, J. B. T., et al. (2016). Methylmercury and brain development: a review of recent literature. J. Trace Elem. Med. Biol. 38, 99-107. doi: 10.1016/j.jtemb.2016.03.001

Beer, Z., Vavra, P., Atucha, E., Rentzing, K., Heinze, H. J., and Sauvage, M. M. (2018). The memory for time and space differentially engages the proximal and distal parts of the hippocampal subfields CA1 and CA3. PLoS Biol. 16:e2006100. doi: 10.1371/journal.pbio.2006100

Bergstrom, K. S. B., Kissoon-Singh, V., Gibson, D. L., Ma, C., Montero, M., Sham, H. P., et al. (2010). Muc2 protects against lethal infectious colitis by 
disassociating pathogenic and commensal bacteria from the colonic mucosa. PLoS Pathog. 6:e1000902. doi: 10.1371/journal.ppat.1000902

Bhat, M., Pasini, E., Copeland, J., Angeli, M., Husain, S., Kumar, D., et al. (2017). Impact of immunosuppression on the metagenomic composition of the intestinal microbiome: a systems biology approach to post-transplant diabetes. Sci Rep. 7:10277. doi: 10.1038/s41598-017-10471-2

Boldrini, M., Fulmore, C. A., Tartt, A. N., Simeon, L. R., Pavlova, I., Poposka, V., et al. (2018). Human hippocampal neurogenesis persists throughout aging. Cell Stem Cell 22, 589-599. doi: 10.1016/j.stem.2018.03.015

Bose, R., Onishchenko, N., Edoff, K., Janson Lang, A. M., and Ceccatelli, S. (2012). Inherited effects of low-dose exposure to methylmercury in neural stem cells. Toxicol. Sci. 130, 383-390. doi: 10.1093/toxsci/kfs 257

Calabrese, V., Cornelius, C., Dinkova-Kostova, A. T., Calabrese, E. J., and Mattson, M. P. (2010). Cellular stress responses, the hormesis paradigm, and vitagenes: novel targets for therapeutic intervention in neurodegenerative disorders. Antioxid. Redox Signal. 13, 1763-1811. doi: 10.1089/ars.2009.3074

Cerdó, T., Diéguez, E., and Campoy, C. (2020). Impact of gut microbiota on neurogenesis and neurological diseases during infancy. Curr. Opin. Pharmacol. 50, 33-37. doi: 10.1016/j.coph.2019.11.006

Clarkson, T. W., and Magos, L. (2006). The toxicology of mercury and its chemical copounds. Crit. Rev. Toxicol. 36, 609-662. doi: 10.1080/10408440600845619

Cohen, S., Ke, X., Liu, Q., Fu, Q., Majnik, A., and Lane, R. (2016). Adverse early life environment increases hippocampal microglia abundance in conjunction with decreased neural stem cells in juvenile mice. Int. J. Dev. Neurosci. 55, 56-65. doi: 10.1016/j.ijdevneu.2016.09.010

Dunphy-Doherty, F., O’Mahony, S. M., Peterson, V. L., O'Sullivan, O., Crispie, F., Cotter, P. D., et al. (2018). Post-weaning social isolation of rats leads to long-term disruption of the gut microbiota-immune-brain axis. Brain Behav. Immun. 68, 261-273. doi: 10.1016/j.bbi.2017.10.024

Falluel-Morel, A., Sokolowski, K., Sisti, H. M., Zhou, X., Shors, T. J., and DiCiccoBloom, E. (2007). Developmental mercury exposure elicits acute hippocampal cell death, reductions in neurogenesis, and severe learning deficits during puberty. J. Neurochem. 103, 1968-1981. doi: 10.1111/j.1471-4159.2007.04882.x

García-Domínguez, I., Veselá, K., García-Revilla, J., Carrillo-Jiménez, A., RocaCeballos, M. A., Santiago, M., et al. (2018). Peripheral inflammation enhances microglia response and nigral dopaminergic cell death in an in vivo MPTP model of parkinson's disease. Front. Cell. Neurosci. 12:398. doi: $10.3389 /$ fncel.2018.00398

Hoffman, J. D., Parikh, I., Green, S. J., Chlipala, G., Mohney, R. P., Keaton, M., et al. (2017). Age drives distortion of brain metabolic, vascular and cognitive functions, and the gut microbiome. Front. Aging Neurosci. 9:298. doi: $10.3389 /$ fnagi.2017.00298

Holota, Y., Dovbynchuk, T., Kaji, I., Vareniuk, I., Dzyubenko, N., Chervinska, T., et al. (2019). The long-term consequences of antibiotic therapy: role of colonic short chain fatty acids (SCFA) system and intestinal barrier integrity. PLoS ONE 14:e0220642. doi: 10.1371/journal.pone.0220642

Indiani, C. M. D. S.P., Rizzardi, K. F., Castelo, P. M., Ferraz, L. F. C., Darrieux, M., and Parisotto, T. M. (2018). Childhood obesity and firmicutes/bacteroidetes ratio in the gut microbiota: a systematic review. Child. Obes. 14, 501-509. doi: $10.1089 /$ chi.2018.0040

Jiang, X., Gu, S., Liu, D., Zhao, L., Xia, S., He, X., et al. (2018). Lactobacillus brevis 23017 relieves mercury toxicity in the colon by modulation of oxidative stress and inflammation through the interplay of MAPK and NF-кB signaling cascades. Front. Microbiol. 9:02425. doi: 10.3389/fmicb.2018.02425

Kempermann, G., Song, H., and Gage, F. H. (2015). Neurogenesis in the adult hippocampus. Cold Spring Harb Perspect Biol. 7:a018812. doi: $10.1101 /$ cshperspect.a018812

Kim, W., Yoo, D. Y., Jung, H. Y., Kim, J. W., Hahn, K. R., Kwon, H. J., et al. (2019). Leaf extracts from dendropanax morbifera Léveille mitigate mercury-induced reduction of spatial memory, as well as cell proliferation, and neuroblast differentiation in rat dentate gyrus. BMC Complement Altern. Med. 19:94. doi: 10.1186/s12906-019-2508-6

Lebel, C., and Deoni, S. (2018). The development of brain white matter microstructure neuroimage 182, 207-218. doi: 10.1016/j.neuroimage.2017.12.097

Leocádio, P. C. L., Oriá, R. B., Crespo-Lopez, M. E., and Alvarez-Leite, J. I. (2020). Obesity: more than an inflammatory, an infectious disease? Front. Immunol. 10:3092. doi: 10.3389/fimmu.2019.03092
Leri, M., Scuto, M., Ontario, M. L., Calabrese, V., Calabrese, E. J., Bucciantini, M., et al. (2020). Healthy effects of plant polyphenols: molecular mechanisms. Int. J. Mol. Sci. 21:1250. doi: 10.3390/ijms21041250

Lin, X., Zhao, J., Zhang, W., He, L., Wang, L., Chang, D., et al. (2020). Acute oral methylmercury exposure perturbs the gut microbiome and alters gutbrain axis related metabolites in rats. Ecotoxicol. Environ. Saf. 190:110130. doi: 10.1016/j.ecoenv.2019.110130

Lopez, C. A., and Skaar, E. P. (2018). The impact of dietary transition metals on host- bacterial interactions. Cell Host Microbe. 23, 737-748. doi: 10.1016/j.chom.2018.05.008

O'Leary, O. F., Ogbonnaya, E. S., Felice, D., Levone, B. R., Conroy, L. C., Fitzgerald, P., et al. (2018). The vagus nerve modulates BDNF expression and neurogenesis in the hippocampus. Eur. Neuropsychopharmacol. 28, 307-316. doi: 10.1016/j.euroneuro.2017.12.004

Oria, R. B., Empadinhas, N., and Malva, J. O. (2020). Interplay between nutrition, the intestinal microbiota and the immune system. Front. Immunol. 11:1758. doi: $10.3389 /$ fimmu.2020.01758

Oriá, R. B., Malva, J. O., Foley, P. L., Freitas, R. S., Bolick, D. T., and Guerrant, R. L. (2018). Revisiting inbred mouse models to study the developing brain: the potential role of intestinal microbiota. Front. Hum. Neurosci. 12:358. doi: $10.3389 /$ fnhum. 2018.00358

Oriá, R. B., Murray-Kolb, L. E., Scharf, R. J., Pendergast, L. L., Lang, D. R., Kolling, G. L., et al. (2016). Early-life enteric infections: relation between chronic systemic inflammation and poor cognition in children. Nutr. Rev. 74, 374-386. doi: 10.1093/nutrit/nuw008

Pannaraj, P. S., Li, F., Cerini, C., Bender, J. M., Yang, S., Rollie, A., et al. (2017). Association between breast milk bacterial communities and establishment and development of the infant gut microbiome. JAMA Pediatr. 171, 647-654. doi: 10.1001/jamapediatrics.2017.0378

Parks, J. M., Johs, A., Podar, M., Bridou, R., Hurt, R. A., Smith, S. D., et al. (2013). The genetic basis for bacterial mercury methylation. Science 339, 1332-1335. doi: $10.1126 /$ science. 1230667

Rodier, P. M. (1995). Developing brain as a target of toxicity. Environm. Health Perspec. 103, 73-76. doi: 10.1289/ehp.95103s673

Rothenberg, S. E., Keiser, S., Ajami, N. J., Wong, M. C., Gesell, J., Petrosino, J. F., et al. (2016). The role of gut microbiota in fetal methylmercury exposure: insights from a pilot study. Toxicol. Lett. 242, 60-67. doi: $10.1016 /$ j.toxlet.2015.11.022

Ruan, Y., Wu, C., Guo, X., Xu, Z., Xing, C., Cao, H., et al. (2019). High doses of copper and mercury changed cecal microbiota in female mice. Biol. Trace Elem. Res. 189, 134-144. doi: 10.1007/s12011-018-1456-1

Schalk, I. J., Hannauer, M., and Braud, A. (2011). New roles for bacterial siderophores in metal transport and tolerance. Environ. Microbiol. 13, 2844-2854. doi: 10.1111/j.1462-2920.2011.02556.x

Shao, Y., Forster, S. C., Tsaliki, E., Vervier, K., Strang, A., Simpson, N., et al. (2019). Stunted microbiota and opportunistic pathogen colonization in caesareansection birth. Nature 574, 117-121. doi: 10.1038/s41586-019-1560-1

Siblerud, R., Mutter, J., Moore, E., Naumann, J., and Walach, H. (2019). A hypothesis and evidence that mercury may be an etiological factor in alzheimer's disease. Int. J. Environ. Res. Public Health. 16:5152. doi: 10.3390/ijerph16245152

Simões, M. R., Azevedo, B. F., Fiorim, J., Freire, D. D., Covre, E. P., Vassallo, D. V., et al. (2016). Chronic mercury exposure impairs the sympathovagal control of the rat heart. Clin. Exp. Pharmacol. Physiol. 43, 1038-1045. doi: 10.1111/1440-1681.12624

Sokolowski, K., Obiorah, M., Robinson, K., Mccandlish, E., Buckley, B., and Dicicco-Bloom, E. (2013). Neural stem cell apoptosis after low-methylmercury exposures in postnatal hippocampus produce persistent cell loss and adolescent memory deficits. Dev. Neurobiol. 73, 936-949. doi: 10.1002/dneu.22119

Sorrells, S. F., Paredes, M. F., Cebrian-Silla, A., Sandoval, K., Qi, D., Kelley, K. W., et al. (2018). Human hippocampal neurogenesis drops sharply in children to undetectable levels in adults. Nature 555, 377-381. doi: 10.1038/nature25975

Takahashi, T., Fujimura, M., Koyama, M., Kanazawa, M., Usuki, F., Nishizawa, M., et al. (2017). Methylmercury causes blood-brain barrier damage in rats via upregulation of vascular endothelial growth factor expression. PLoS ONE 12:e0170623. doi: 10.1371/journal.pone.0170623

Tran, T. T. T., Corsini, S., Kellingray, L., Hegarty, C., Le Gall, G., Narbad, A., et al. (2019). APOE genotype influences the gut microbiome structure and function 
in humans and mice: relevance for Alzheimer's disease pathophysiology. FASEB J. 33, 8221-8231. doi: 10.1096/fj.201900071R

Valero, J., Mastrella, G., Neiva, I., Sánchez, S., and Malva, J. O. (2014). Long-term effects of an acute and systemic administration of LPS on adult neurogenesis and spatial memory. Front. Neurosci. 8:83. doi: 10.3389/fnins.2014.00083

Wenzel, T. J., Gates, E. J., Ranger, A. L., and Klegeris, A. (2020). Shortchain fatty acids (SCFAs) alone or in combination regulate select immune functions of microglia-like cells. Mol. Cell. Neurosci. 105:103493. doi: 10.1016/j.mcn.2020.103493

Yoshihara, T., Oikawa, Y., Kato, T., Kessoku, T., Kobayashi, T., Kato, S., et al. (2020). The protective effect of bifidobacterium bifidum G9-1 against mucus degradation by akkermansia muciniphila following small intestine injury caused by a proton pump inhibitor and aspirin. Gut Microbes 11, 1385-1404. doi: 10.1080/19490976.2020.1758290

Zhang, B., Bin, L. Y. M., Hu, A. L., Xu, S. H., Fan, L. D., Cheng, M. L., et al. (2019). $\mathrm{HgS}$ and Zuotai differ from $\mathrm{HgCl} 2$ and methyl mercury in intestinal $\mathrm{Hg}$ absorption, transporter expression and gut microbiome in mice. Toxicol. Appl. Pharmacol. 379:114615. doi: 10.1016/j.taap.2019.114615
Zhang, L., Wang, Y., Xiayu, X., Shi, C., Chen, W., Song, N., et al. (2017). Altered gut microbiota in a mouse model of alzheimer's disease. J. Alzheimer's Dis. 60, 1241-1257. doi: 10.3233/JAD-170020

Zhao, Y., Zhou, C., Wu, C., Guo, X., Hu, G., Wu, Q., et al. (2020). Subchronic oral mercury caused intestinal injury and changed gut microbiota in mice. Sci. Total Environ. 721:137639. doi: 10.1016/j.scitotenv.2020.137639

Conflict of Interest: The authors declare that the research was conducted in the absence of any commercial or financial relationships that could be construed as a potential conflict of interest.

Copyright $\odot 2020$ Pinto, Raposo, Matos, Alvarez-Leite, Malva and Oriá. This is an open-access article distributed under the terms of the Creative Commons Attribution License (CC BY). The use, distribution or reproduction in other forums is permitted, provided the original author(s) and the copyright owner(s) are credited and that the original publication in this journal is cited, in accordance with accepted academic practice. No use, distribution or reproduction is permitted which does not comply with these terms. 